\title{
Generalised Solutions of Laplace's Equation
}

By H. S. Rese, Edinburgh University.

(Received 30th November 1930. Read 5th December 1930.)

The present paper contains solutions of the tensor generalisation of Laplace's Equation. The results obtained are summarised in the two theorems enunciated in $\S 1$. They apply only to the case when the Riemannian space forming the background of the theory is flat. In the concluding paragraph a special case is considered, and it is shown that the present theory is closely connected with Whittaker's well known general solution of the ordinary Laplace's Equation. ${ }^{1}$

Let

\section{§1. INTRODUCTION.}

$$
d s^{2}=g_{\mu \nu} d x^{\mu} d x^{\nu}
$$

define the metric of an $n$-dimensional Euclidean space; that is, one for which the Riemann-Christoffel tensor is everywhere zero. Let $\Omega$ be one half of the square of the geodesic distance between the two points $\left(x^{i}\right)$ and $\left(\bar{x}^{i}\right)$ of this space, ${ }^{2}$ so that $\Omega$ is a scalar function of the two sets of variables $\left(x^{i}\right),\left(\bar{x}^{i}\right)$. Let further the coordinates $\left(\bar{x}^{i}\right)$ be each functions of a variable $\tau$. Then $\Omega$ is a function of $x^{1}, x^{2}, \ldots, x^{n}$, and $\tau$, and we shall later define $\tau$ as a function of the $x^{i}$ by means of the equation

$$
\Omega=0 .
$$

Greek suffixes will be used to denote covariant differentiations with respect to the $x^{i}$, with $\tau$ kept constant, the only exception to this rule being that the suffix $\tau$ will denote ordinary partial differentiations with respect to $\tau$. Thus, for example,

$$
\left.\begin{array}{ll}
\Omega_{\mu}=\partial \Omega / \partial x^{\mu}, & \Omega_{\tau}=\partial \Omega / \partial \tau, \\
\Omega_{\mu \nu}=\frac{\partial^{2} \Omega}{\partial x^{\mu} \partial x^{\nu}}-\{\mu \nu, a\} \frac{\partial \Omega}{\partial x^{a}}, & \Omega_{\tau \tau}=\frac{\partial^{2} \Omega}{\partial \tau^{2}}, \\
\Omega_{\tau \mu \nu}=\frac{\partial^{2} \Omega_{\tau}}{\partial x^{\mu} \partial x^{\nu}}-\{\mu \nu, a\} \frac{\partial \Omega_{\tau}}{\partial x^{a}}, & \Omega_{\tau \mu}=\frac{\partial^{2} \Omega}{\partial \tau \partial x^{\mu}},
\end{array}\right\}
$$

1 Whittaker and Watson, "Modern Analysis" (1920), \$18.3.

2 Some properties of this function have been investigated in earlier papers, particularly (i) Proc. London Math. Soc., 31 (1930), 225 ; (ii) ibid., 32 (1931), 87. These will be referred to as papers 1 and 2 respectively. 
and so on. It must be emphasised that in these definitions the partial differentiations with respect to the $x^{i}$ are strict, that is, they treat $\tau$ as a constant as well as the other $x$ 's. The summation convention does not of course hold for the suffix $\tau$.

For convenience the Christoffel symbol $\{\lambda \mu, \nu\}$ will be denoted by $\Gamma_{\lambda \mu}^{\nu}$. Further, the evaluation at $\left(\bar{x}^{i}\right)$ of any function of the $x^{i}$ will be indicated by the superposing of a bar on the functional symbol.

The partial differential equation of which solutions are sought is

$$
V_{\lambda}^{\lambda} \equiv g^{\lambda \mu}\left\{\frac{\partial^{2} V}{\partial x^{\lambda} \partial x^{\mu}}-\Gamma_{\lambda \mu}^{a} \frac{\partial V}{\partial x^{a}}\right\}=0
$$

The following are the theorems proved.

Theorem I. If the functions $\bar{x}^{i}(\tau)$ are chosen to satisfy the differential equations

$$
\begin{array}{r}
\bar{g}_{\mu \nu} \frac{d \bar{x}^{\mu}}{d \tau} \frac{d \bar{x}^{\nu}}{d \tau}=0, \\
\frac{d^{2} \bar{x}^{\mu}}{d \tau^{2}}+\bar{\Gamma}_{a \beta}^{\mu} \frac{d \bar{x}^{a}}{d \tau} \frac{d \bar{x}^{\beta}}{d \tau}=0,
\end{array}
$$

then, for all values of $n, a$ solution of the partial differential equation $V_{\lambda}^{\lambda}=0$ is given by

$$
V=f\left(\Omega_{\tau}\right)
$$

where, after differentiating, ${ }^{1} \tau$ is expressed as a function of $x^{1}, x^{2}, \ldots, x^{n}$ by means of the equation $\Omega=0$, and where $f\left(\Omega_{\tau}\right)$ is an arbitrary function of $\Omega_{\tau}$.

That the equations (1.5) and (1.6) are compatible is well known. ${ }^{2}$ TheOREM II. A solution of the equation $V_{\lambda}^{\lambda}=0$ is given by

$$
V=\phi(\tau) / \Omega_{\tau} t^{(n-2)},
$$

where $\phi(\tau)$ is an arbitrary function of $\tau$, and $\tau$ is expressed as a function of the $x$ 's by means of the equation $\Omega=0$. If the number $n$ of the

$1 \partial \Omega / \partial \tau$ is in general a function of $\tau$ as well as of the $x$ 's, so $\tau$ must be eliminated in order that the solution should be expressed as a function of the $x$ 's only.

2 See, for example, Veblen, "Invariants of Quadratic Differential Forms" Camb.). Math. Tract. No. 24) (1927), 95. 
rariables is equal to 2 or 4 there is no limitation on the choice of the functions $\bar{x}^{i}(\tau)$; but if $n$ has any other value, these functions must satisfy the conditions (1.5) and (1.6).

\section{§2. Preliminary Formulae.}

It is well known that $\Omega$ satisfies ${ }^{1}$ the partial differential equation where $\Omega^{\lambda}=g^{\lambda a} \Omega_{a}$.

$$
\Omega^{\lambda} \Omega_{\lambda}=2 \Omega,
$$

Moreover, it is shown elsewhere ${ }^{2}$ that, the space being flat,

$$
\Omega_{\mu \nu}=g_{\mu \nu} .
$$

Furthermore, since we have put

$$
\Omega=0
$$

it follows, by differentiating partially with respect to $x^{\lambda}$, that

$$
\Omega_{\lambda}+\Omega_{\tau} \tau_{\lambda}=0,
$$

where $\tau_{\lambda}=\partial \tau / \partial x^{\lambda}$. Hence

$$
\tau_{\lambda}=-\Omega_{\lambda} / \Omega_{\tau} .
$$

Differentiating (2.1) twice in succession with respect to $\tau$, we get

$$
\begin{aligned}
\Omega_{\tau \lambda} \Omega^{\lambda} & =\Omega_{\tau}, \\
\Omega_{\tau \tau \lambda} \Omega^{\lambda}+\Omega_{\tau \lambda} \Omega_{\tau}^{\lambda} & =\Omega_{\tau \tau} .
\end{aligned}
$$

From (2.2), raising the suffix $\nu$ and contracting,.

$$
\Omega_{\mu}^{\mu}=n,
$$

and the differentiation of this equation with respect to $\tau$ gives

$$
\Omega_{\tau \mu}^{\mu}=0 .
$$

By (2.1), (2.3), (2.4), it follows that

$$
\tau^{\lambda} \tau_{\lambda}=0 .
$$

By (2.4),

$$
\begin{aligned}
\tau_{\lambda}^{\lambda} & =g_{\sigma}^{\lambda \tau} \tau_{\lambda a}=-g^{\lambda a}\left[\left(\frac{\Omega_{\lambda}}{\Omega_{\tau}}\right)_{a}+\left(\frac{\Omega_{\lambda}}{\Omega_{\tau}}\right)_{\tau} \tau_{a}\right] \\
& =\Omega_{\tau}^{-2}\left(\Omega_{\tau}^{\lambda} \Omega_{\lambda}-\Omega_{\tau} \Omega_{\lambda}^{\lambda}-\Omega_{\tau} \Omega_{\tau \lambda} \tau^{\lambda}+\Omega_{\tau \tau} \Omega_{\lambda} \tau^{\lambda}\right),
\end{aligned}
$$

whence, by $(2.4),(2.5),(2.7)$ and $(2.9)$,

$$
\tau_{\lambda}^{\lambda}=-(n-2) \Omega_{\tau}^{-1} .
$$

1 This in fact follows at once from equations (1) and (10) of paper 2.

2 Paper $1, \S 2$, where $\Omega$ denotes twice the function here represented by $\Omega$. 
Lastly, it follows from (2.2), by interchanging the $x$ 's and the$\bar{x}$ 's, that

$$
\frac{\partial^{2} \Omega}{\partial \bar{x}^{\mu} \partial \tilde{x}^{\nu}}-\bar{\Gamma}_{\mu \nu}^{a} \frac{\partial \Omega}{\partial \bar{x}^{\alpha}}=\dot{g}_{\mu \nu}
$$

But

$$
\frac{\partial \Omega}{\partial \tau}=\frac{\partial \Omega}{\partial \bar{x}^{a}} \frac{\partial \bar{x}^{a}}{\partial \tau}
$$

and

$$
\frac{\partial^{2} \Omega}{\partial \tau^{2}}=\frac{\partial \Omega}{\partial \bar{x}^{a}} \frac{d^{2} \bar{x}^{a}}{d \tau^{2}}+\frac{\partial^{2} \Omega}{\partial \bar{x}^{a} \partial \bar{x}^{\beta}} \frac{d \bar{x}^{a}}{d \tau} \frac{d \bar{x}^{\beta}}{d \tau}
$$

and therefore, by (2.11),

$$
\Omega_{\tau \tau}=\bar{g}_{\mu \nu} \frac{d \bar{x}^{\mu}}{d \tau} \frac{d \bar{x}^{\nu}}{d \tau}+\frac{\partial \Omega}{\partial \bar{x}^{\alpha}}\left(\frac{d^{2} \bar{x}^{\alpha}}{d \tau^{2}}+\bar{\Gamma}_{\mu \nu}^{a} \frac{d \bar{x}^{\mu}}{d \tau} \frac{d \bar{x}^{\nu}}{d \tau}\right) .
$$

\section{§3. Proof of Theorem I.}

We now show, by direct substitution, that any function of $\Omega_{\tau}$, say

$$
U=f\left(\Omega_{\tau}\right)
$$

is a solution of the equation

$$
V_{\lambda}^{\lambda}=0
$$

provided that the conditions (1.5) and (1.6) are satisfied.

For

and

$$
U_{\lambda}=f^{\prime}\left(\Omega_{\tau}\right)\left(\Omega_{\tau \lambda}+\Omega_{\tau \tau} \tau_{\lambda}\right),
$$

$$
\begin{aligned}
& U_{\lambda}^{\lambda}=f^{\prime \prime}\left(\Omega_{\tau}\right)\left(\Omega_{\tau \lambda}+\Omega_{\tau \tau} \tau_{\lambda}\right)\left(\Omega_{\tau}^{\lambda}+\Omega_{\tau \tau} \tau^{\lambda}\right) \\
&+f^{\prime}\left(\Omega_{\tau}\right)\left(\Omega_{\tau \lambda}^{\lambda}+2 \Omega_{\tau \tau \lambda} \tau^{\lambda}+\Omega_{\tau \tau \tau} \tau_{\lambda} \tau^{\lambda}+\Omega_{\tau \tau} \tau_{\lambda}^{\lambda}\right) \\
&=f^{\prime \prime}\left(\Omega_{\tau}\right)\left(\Omega_{\tau \lambda} \Omega_{\tau}^{\lambda}+2 \Omega_{\tau \tau} \Omega_{\tau}^{\lambda} \tau_{\lambda}\right)+f^{\prime}\left(\Omega_{\tau}\right)\left(\Omega_{\tau \lambda}^{\lambda}+2 \Omega_{\tau \tau \lambda} \tau^{\lambda}+\Omega_{\tau \tau} \tau_{\lambda}^{\lambda}\right),
\end{aligned}
$$

using equation (2.9).

By (2.4), (2.5), (2.6), (2.8) and (2.10), it quickly follows that

$$
U_{\lambda}^{\lambda}=-f^{\prime \prime}\left(\Omega_{\tau}\right)\left(\Omega_{\tau \tau \lambda} \Omega^{\lambda}+\Omega_{\tau \tau}\right)-\Omega_{\tau}^{-1} f^{\prime}\left(\Omega_{\tau}\right)\left\{2 \Omega_{\tau \tau \lambda} \Omega^{\lambda}+(n-2) \Omega_{\tau \tau}\right\} .
$$

By (2.12), if the functions $\bar{x}^{i}(\tau)$ satisfy the relations

$$
\begin{gathered}
\bar{g}_{\mu \nu} \frac{d \bar{x}^{\mu}}{d \tau} \frac{d \bar{x}^{\dot{\nu}}}{d \tau}=0, \\
\frac{d^{2} \bar{x}^{\mu}}{d \tau^{2}}+\bar{\Gamma}_{\alpha \beta}^{\mu} \frac{d \bar{x}^{\alpha}}{d \tau} \frac{d \bar{x}^{\beta}}{d \tau}=0,
\end{gathered}
$$

then

$$
\Omega_{\tau \tau}=0 \text {. }
$$


Differentiating with respect to $x^{\lambda}$,

and therefore

$$
\Omega_{\tau \tau \lambda}+\Omega_{\tau \tau \tau} \tau_{\lambda}=0,
$$

$$
\Omega_{\tau \lambda \lambda} \Omega^{\lambda}=0,
$$

since $\Omega^{\lambda} \tau_{\lambda}=0$ by (2.4) and (2.9).

By (3.4), (3.7), (3.8) it follows that

$$
U_{\lambda}^{\lambda}=0
$$

provided that the conditions (3.5) and (3.6) are satisfied. This. concludes the proof of Theorem I.

\section{§4. Proof of Theorem II.}

The next problem is to show that, if

$$
V=\phi(\tau) / \Omega_{\tau}^{\frac{\hbar}{(}(n-2)},
$$

where $\phi(\tau)$ is an arbitrary function of $\tau$, then $V$ satisfies the equation $V_{\lambda}^{\lambda}=0$. It will be shown that the restrictions (3.5) and (3.6) must still be imposed except when $n=2$ or $n=4$.

Consider first the function $W$ defined by

$$
W=\Omega_{\tau}^{-\frac{1}{2}(n-2)} \text {. }
$$

Putting $f\left(\Omega_{\tau}\right)=\Omega_{\tau}^{-\frac{1}{2}(n-2)}$ in (3.3) and (3.4),

$$
\begin{aligned}
& W_{\lambda}=-\frac{1}{2}(n-2) \Omega_{\tau}^{-\frac{1}{2} n}\left(\Omega_{\tau \lambda}+\Omega_{\tau \tau} \tau_{\lambda}\right), \\
& W_{\lambda}^{\lambda}=\frac{1}{4}(n-2)(n-4) \Omega_{\tau}^{-\frac{1}{2(n+2)}}\left(\Omega_{\tau \tau}-\Omega_{\tau \tau \lambda} \Omega_{1}^{\lambda}\right) .
\end{aligned}
$$

Hence, if $n=2$ or $n=4$, we have

$$
W_{\lambda}^{\lambda}=0
$$

without any restrictions being placed on the choice of the functions $\bar{x}^{i}(\tau)$. But, if $n$ has neither of these values, it follows as in the previous paragraph that we shall still have

$$
W_{\lambda}^{\lambda}=0
$$

provided that the $\bar{x}^{i}(\tau)$ satisfy the relations

$$
\begin{aligned}
& \dot{g}_{\mu \nu} \frac{d \bar{x}^{\mu}}{d \tau} \frac{d \bar{x}^{\nu}}{d \tau}=0, \\
& \frac{d^{2} \vec{x}^{\mu}}{d \tau^{2}}+\bar{\Gamma}_{\alpha \beta}^{\mu} \frac{d \bar{x}^{\alpha}}{d \tau} \frac{d \bar{x}^{\beta}}{d \tau}=0 .
\end{aligned}
$$


Now consider the function $V$ defined by

$$
V=W \phi(\tau) \text {, }
$$

where $\phi(\tau)$ is any function of $\tau$. Covariant differentiation gives

$$
\begin{aligned}
V_{\lambda} & =W_{\lambda} \phi(\tau)+W \tau_{\lambda} \phi^{\prime}(\tau), \\
V_{\lambda}^{\lambda} & =W_{\lambda}^{\lambda} \phi(\tau)+2 W_{\lambda} \tau^{\lambda} \phi^{\prime}(\tau)+W \tau_{\lambda} \tau^{\lambda} \phi^{\prime \prime}(\tau)+W \tau_{\lambda}^{\lambda} \phi^{\prime}(\tau) \\
& =\phi^{\prime}(\tau)\left(2 W_{\lambda} \tau^{\lambda}+W \tau_{\lambda}^{\lambda}\right),
\end{aligned}
$$

by $(4.5),(4.5)^{*}$ and $(2.9)$.

Equation (4.8) therefore gives a solution of $V_{\lambda}^{\lambda}=0$ provided that

$$
2 W_{\lambda} \tau^{\lambda}+W \tau_{\lambda}^{\lambda}=0
$$

But by (4.3), (4.2) and (2.10), the left-hand side of this equation is equal to

$$
-(n-2) \Omega_{\tau}^{-\frac{1-1 n}{2}}\left(\Omega_{\tau \lambda} \tau^{\lambda}+\Omega_{\tau \tau} \tau_{\lambda} \tau^{\lambda}\right)-(n-2) \Omega_{\tau}^{-\frac{1}{2} n},
$$

which is zero in virtue of equations (2.4), (2.5) and (2.9), for all values of $n$. We deduce finally, therefore, that

$$
V=\phi(\tau) / \Omega_{\tau}^{\ddagger(-2)}
$$

is a solution of $V_{\lambda}^{\lambda}=0$ provided that, when $n$ has a value other than 2 or 4 , the choice of the functions $x^{i}(\tau)$ is restricted by the equations (4.6) and (4.7).

When $n=2$ this theorem is the tensor generalisation of the well-known fact that any function of $x \pm i y$ is a solution of the equation $\frac{\partial^{2} V}{\partial x^{2}}+\frac{\partial^{2} V}{\partial y^{2}}=0$. When $n=4$ it gives a generalisation of a solution, due to Conway, of the classical wave-equation of mathematical physics. ${ }^{1}$

\section{§5. Connection with WhittakeR's Soldtion of Laplace's Equation.}

Apply Theorem $I$ to the case when $n=3$ and the metric is given by

$$
d s^{2}=d x^{2}+d y^{2}+d z^{2} ; \quad\left(x^{1}=x, x^{2}=y, x^{3}=z\right)
$$

I See Bateman, “Electrical and Optical Wave Motion” (1915), 115. 
The equation to be solved is then the ordinary Laplace's Equation

$$
\frac{\partial^{2} V}{\partial x^{2}}+\frac{\partial^{2} V}{\partial y^{2}}+\frac{\partial^{2} V}{\partial z^{2}}=0
$$

Also, of course,

$$
2 \Omega=(x-\bar{x})^{2}+(y-\bar{y})^{2}+(z-\bar{z})^{2} .
$$

The restrictions (4.6) and (4.7) placed on the choice of $\bar{x}, \bar{y}, \bar{z}$ as functions of $\tau$ reduce in this case to

$$
\left(\frac{d \bar{x}}{d \bar{\tau}}\right)^{2}+\left(\frac{d \bar{y}}{d \tau}\right)^{2}+\left(\frac{d \bar{z}}{d \tau}\right)^{2}=0
$$

and

$$
\frac{d^{2} \bar{x}}{d \tau^{2}}=0=\frac{d^{2} \bar{y}}{d \tau^{2}}=\frac{d^{2} \bar{z}}{d \tau^{2}} \text {. }
$$

The most general solutions of (5.3) and (5.4) are

$$
\left.\begin{array}{l}
\bar{x}=a+\lambda i \tau \cos u \\
\bar{y}=b+\lambda i \tau \sin u \\
\bar{z}=c+\lambda \tau
\end{array}\right\}
$$

where $i=\sqrt{-1}$ and $a, b, c, \lambda, u$ are arbitrary constants. Take $a=b=c=0$ and $\lambda=-1$. Substituting from (5.5) in (5.2), we have

$$
2 \Omega=r^{2}+2 \tau(i x \cos u+i y \sin u+z),
$$

where

$$
r^{2}=x^{2}+y^{2}+z^{2}
$$

and hence

$$
\Omega_{\tau}=i x \cos u+i y \sin u+z .
$$

A solution of equation (5.1) is therefore, by Theorem I,

$$
V=f(i x \cos u+i y \sin u+z, u),
$$

where $f$ is an arbitrary function and $u$ an arbitrary constant. ${ }^{1}$ It follows therefore that

$$
\int f(i x \cos u+i y \sin u+z, u) d u
$$

is also a solution of (5.1), provided that the limits of integration are such that differentiation under the integral sign is permissible.

Since $u$ is an arbitrary constant, the function $f$ of the two arguments $i x \cos u+i y \sin u+z$ and $u$, is (regarded as a function of $x, y, z$ ), an arbitrary function of the former argument only; that is, of $\partial \Omega / \partial \tau$ only. 
Whittaker has shown ${ }^{1}$ that the most general solution of Laplace's Equation is of this form.

An application of Theorem II to the same special case leads ultimately to the conclusion that the integral

$$
\int \frac{1}{r} \psi\left(\frac{i x \cos u+i y \sin u+z}{r^{2}}, u\right) d u
$$

gives a solution of (5.1), $\psi$ being an arbitrary function of its arguments. It is however a well known fact that if a function $\chi(x, y, z)$ satisfies Laplace's Equation, so also does $\frac{1}{r} \chi\left(\frac{x}{r^{2}}, \frac{y}{r^{2}}, \frac{z}{r^{2}}\right)$. The solution (5.8) is therefore deducible from (5.7).

1 Whittaker and Watson, loc. cit. 